\title{
Region of Interest Reconstruction From Truncated Data in Circular Cone-Beam CT
}

\author{
Lifeng Yu, Yu Zou, Emil Y. Sidky, Charles A. Pelizzari, Peter Munro, and Xiaochuan Pan*, Senior Member, IEEE
}

\begin{abstract}
The circular scanning trajectory is one of the most widely adopted data-acquisition configurations in computed tomography (CT). The Feldkamp, Davis, Kress (FDK) algorithm and its various modifications have been developed for reconstructing approximately three-dimensional images from circular cone-beam data. When data contain transverse truncations, however, these algorithms may reconstruct images with significant truncation artifacts. It is of practical significance to develop algorithms that can reconstruct region-of-interest (ROI) images from truncated circular cone-beam data that are free of truncation artifacts and that have an accuracy comparable to that obtained from nontruncated cone-beam data. In this work, we have investigated and developed a backprojection-filtration (BPF)-based algorithm for ROI-image reconstruction from circular cone-beam data containing transverse truncations. Furthermore, we have developed a weighted BPF algorithm to exploit "redundant" information in data for improving image quality. In an effort to validate and evaluate the proposed $B P F$ algorithms for circular cone-beam CT, we have performed numerical studies by using both computer-simulation data and experimental data acquired with a radiotherapy cone-beam CT system. Quantitative results in these studies demonstrate that the proposed BPF algorithms for circular cone-beam CT can reconstruct ROI images free of truncation artifacts.
\end{abstract}

Index Terms-Computed tomography (CT), cone-beam CT, image reconstruction, region of interest (ROI), truncated projection.

\section{INTRODUCTION}

C IRCULAR scanning trajectory has been widely used in computed tomography (CT) for data acquisition because it involves minimum hardware implementation complexity. It has found important applications in micro-CT [1]-[3], dedicated breast CT [4]-[6], and radiotherapy CT [7]-[10]. The circular cone-beam configuration does not, however, satisfy Tuy's sufficiency condition [11], [12], and no stable algorithm exists for exact reconstruction of three-dimensional (3-D) images from circular cone-beam projections of a longitudinally nonuniform object function. The so-called Feldkamp, Davis, Kress (FDK) algorithm [13] and other modifications [14]-[18] have been developed for reconstructing approximately $3-\mathrm{D}$ images from cir-

Manuscript received October 3, 2005; revised January 20, 2006. This work was supported by the National Institutes of Health under Grant EB00225 and Grant EB02765. The work of E. Y. Sidky was supported by the National Institutes of Health under Grant EB003913. Asterisk indicates corresponding author.

L. Yu, Y. Zou, and E. Y. Sidky are with the Department of Radiology, The University of Chicago, Chicago, IL 60637 USA.

C. A. Pelizzari is with Department of Radiation and Cellular Oncology, The University of Chicago, Chicago, IL 60637 USA

P. Munro is with Varian Medical Systems, Palo Alto, CA 94304 USA.

*X. Pan is with the Department of Radiology, The University of Chicago, Chicago, IL 60637 USA (e-mail: xpan@uchicago.edu).

Digital Object Identifier 10.1109/TMI.2006.872329 cular cone-beam data. For a relatively small cone angle and certain object functions, these algorithms can yield images that are adequately accurate for some practical applications.

In imaging tasks involving micro-CT, dedicated breast $\mathrm{CT}$, and radiotherapy megavoltage (MV) and KV kilovoltage (KV) $\mathrm{CT}$, it is not uncommon that the field of view (FOV) of the CT system is smaller than the support of the object function. Also, in these applications, one is often interested in information within a region of interest (ROI) of the subject; and, for the sake of reducing radiation dose delivered to the subject, one may intend to use an FOV that fully covers the ROI and that is otherwise considerably smaller than the subject. These situations can lead to transversely truncated projection data from which the existing algorithms [13]-[18] generally yield images of the subject with severe artifacts. On the other hand, it is of practical significance to develop algorithms that can, from truncated circular cone-beam data, reconstruct ROI images with an accuracy comparable to that obtained from nontruncated data. In this work, we focus on investigating and developing such algorithms for ROI-image reconstruction from circular cone-beam data containing transverse truncations.

Recently, an algorithm has been proposed for exact image reconstruction on PI-lines from helical cone-beam data [19], [20] This algorithm, which we refer to as the backprojection-filtration (BPF) algorithm, reconstructs images by first computing the cone-beam backprojection of the data derivatives onto PI-lines and then performing a one-dimensional (1-D) filtering of the backprojection image along PI-lines. The BPF algorithm can exactly reconstruct images by use of data less than that required by other existing filtered-backprojection (FBP)-based algorithms, such as Katsevich's algorithm [21]. It has also been shown explicitly that the BPF algorithm can reconstruct an ROI image within the subject from fan-beam data containing truncations [22]-[24].

In this work, based upon the BPF algorithm for helical conebeam CT, we develop an approximate BPF algorithm for 3-D ROI-image reconstruction from circular cone-beam data containing truncations. We show that this algorithm, which is referred to as the BPF algorithm for circular cone-beam CT, can reconstruct exactly an ROI image within the midplane and approximate ROI images within off-midplanes and that these ROI images are free of truncation artifacts in images obtained with the FDK algorithms. Furthermore, we generalize this approximate BPF algorithm to exploit "redundant" information in data for further improving image quality. In addition to computersimulation studies, we also apply the proposed algorithm to reconstructing ROI images from circular cone-beam data acquired with a simulator-CT system for radiotherapy (Acuity, Varian Medical Systems). 


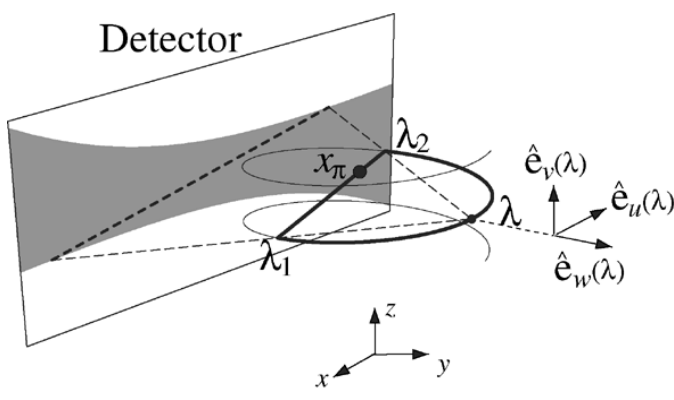

Fig. 1. Illustration of the helical cone-beam scanning geometry and a PI-line segment. Rotation-coordinate system whose origin is fixed on the source point $\lambda$ is specified by three unit vectors $\hat{\mathrm{e}}_{u}, \hat{\mathrm{e}}_{v}$, and $\hat{\mathrm{e}}_{w}$. PI-line segment jointing two points on the helix is labeled by $\lambda_{1}$ and $\lambda_{2}$, where $\left|\lambda_{2}-\lambda_{1}\right| \leq 2 \pi$; and $x_{\pi}$ denotes a point on this PI-line segment.

The paper is organized as follows. In Section II, following a brief review of the BPF algorithm for helical cone-beam CT, we derive an approximate BPF algorithm for circular cone-beam $\mathrm{CT}$ and subsequently generalize it to incorporate data redundancy information. In Section III, we present results of our numerical studies by using both computer-simulation data and experimental data for validation and evaluation of the proposed BPF algorithm for circular cone-beam CT. In the experimental study, we acquired circular cone-beam data by using a simulator-CT system for radiation therapy. Finally, we make remarks and conclusions in Section IV.

\section{THEORY}

\section{A. BPF Algorithm for Helical Cone-Beam CT}

We first briefly review the BPF algorithm for helical conebeam CT [19], [20]. In a helical cone-beam scan, the imaged object is translated longitudinally while the X-ray source and detector rotate. Consider a coordinate system $\{x, y, z\}$ that is fixed on the imaged object. As shown in Fig. 1, from the perspective of the object, the source trajectory is a helix and can be written in the fixed-coordinate system as

$$
\vec{r}_{0}(\lambda)=\left(R \cos \lambda, R \sin \lambda, \frac{h}{2 \pi} \lambda\right)^{T}
$$

where $\lambda$ denotes the rotation angle of the $\mathrm{X}$-ray source, $R$ the distance from the source point to the rotation axis, and $h$ the pitch of the helical trajectory, which is defined as the translation distance of the imaged object during one turn of gantry rotation. The cylindrical volume enclosed by the helical trajectory is referred to as the helix cylinder, and we assume that the support of the object function $f(\vec{r})$ is within the helix cylinder.

We introduce a rotation-coordinate system whose origin is fixed on the source point. In the fixed-coordinate system, the three unit vectors of the rotation-coordinate system can be written as

$$
\begin{aligned}
\hat{\mathrm{e}}_{u} & =(-\sin \lambda, \cos \lambda, 0)^{T} \\
\hat{\mathrm{e}}_{v} & =(0,0,1)^{T} \\
\hat{\mathrm{e}}_{w} & =(\cos \lambda, \sin \lambda, 0)^{T} .
\end{aligned}
$$

Without loss of generality, we consider a flat-panel detector, which has a normal direction along $\hat{\mathrm{e}}_{w}$ and is at a distance $S$ from the source point. Any point on the detector can be specified by two parameters $u$ and $v$. At angle $\lambda$, the cone-beam projection of the object function $f(\vec{r})$ at a point $(u, v)$ on the detector can be expressed as

$$
P(u, v, \lambda)=\int_{0}^{\infty} d s f\left(\vec{r}_{0}(\lambda)+s \hat{\beta}\right)
$$

where the unit vector $\hat{\beta}$ indicates the direction of the ray starting from source point $\vec{r}_{0}(\lambda)$ and passing through the point $(u, v)$ on the detector and can be written as

$$
\hat{\beta}=\frac{1}{\sqrt{u^{2}+v^{2}+S^{2}}}\left[u \hat{\mathrm{e}}_{u}(\lambda)+v \hat{\mathrm{e}}_{v}(\lambda)-S \hat{\mathrm{e}}_{w}(\lambda)\right] .
$$

A PI-line is a straight line intersecting with the helical trajectory at two points labeled by $\lambda_{1}$ and $\lambda_{2}$, where $\left|\lambda_{2}-\lambda_{1}\right| \leq 2 \pi$; and the segment on the PI-line within the helix cylinder is referred to as the PI-line segment. We use

$$
\hat{\mathrm{e}}_{\pi}=\frac{\vec{r}_{0}\left(\lambda_{2}\right)-\vec{r}_{0}\left(\lambda_{1}\right)}{\left|\overrightarrow{r_{0}}\left(\lambda_{2}\right)-\vec{r}_{0}\left(\lambda_{1}\right)\right|}
$$

to denote the direction of the PI-line segment and $x_{\pi}$ to index a point on the PI-line segment. It has been shown that $\left(x_{\pi}, \lambda_{1}, \lambda_{2}\right)$ can specify a unique point within the helix cylinder and any point within the helix cylinder can be uniquely described by $\left(x_{\pi}, \lambda_{1}, \lambda_{2}\right)$ [25]. Therefore, $\left\{x_{\pi}, \lambda_{1}, \lambda_{2}\right\}$ can be referred to as the PI-line coordinate system. For a point $\vec{r}$ within the helix cylinder, the relation between PI-line coordinates and $\vec{r}$ is given by

$$
\vec{r}=\vec{r}_{c 0}+x_{\pi} \hat{\mathrm{e}}_{\pi}
$$

where $\vec{r}_{c 0}=\left(\vec{r}_{0}\left(\lambda_{1}\right)+\vec{r}_{0}\left(\lambda_{2}\right)\right) / 2, x_{\pi} \in\left(x_{\pi 1}, x_{\pi 2}\right)$, and $x_{\pi 1}$ and $x_{\pi 2}$ denote the two end points of a PI-line segment. Moreover, we use $x_{s 1}$ and $x_{s 2}$ to denote the two end points of the intersection of the PI-line segment with the support of the object function. We refer to this intersection as the support segment on the PI-line. Because the object function is enclosed completely within the helix cylinder, we have $\left[x_{\pi 1}, x_{\pi 2}\right] \supset\left[x_{s 1}, x_{s 2}\right]$.

The concept of PI-line segment plays an important role in the development of algorithms for image reconstruction in helical cone-beam CT [19], [25]-[27]. It is clear that a given point $\vec{r}$ within the helix determines a unique PI-line segment and that the PI-line segments can completely fill the helix cylinder [25]. Therefore, the image reconstruction within the helix cylinder is equivalent to the image reconstruction on PI-line segments. Let $f_{\pi}\left(x_{\pi}, \lambda_{1}, \lambda_{2}\right)$ denote the image function on a PI-line segment, which can be reconstructed by use of the BPF algorithm as [19], [20], [28]

$$
\begin{aligned}
& f_{\pi}\left(x_{\pi}, \lambda_{1}, \lambda_{2}\right) \\
&= \frac{1}{2 \pi^{2}} \frac{1}{\sqrt{\left(x_{B}-x_{\pi}\right)\left(x_{\pi}-x_{A}\right)}} \\
& \quad \times\left[\int_{x_{A}}^{x_{B}} \frac{d x_{\pi}^{\prime}}{x_{\pi}-x_{\pi}^{\prime}} \sqrt{\left(x_{B}-x_{\pi}^{\prime}\right)\left(x_{\pi}^{\prime}-x_{A}\right)}\right. \\
&\left.\quad \times g_{\pi}\left(x_{\pi}^{\prime}, \lambda_{1}, \lambda_{2}\right)+2 \pi P_{0}\left(u_{\pi}, v_{\pi}, \lambda_{1}\right)\right]
\end{aligned}
$$


where $x_{A}$ and $x_{B}$ are any points satisfying $\left[x_{\pi 1}, x_{\pi 2}\right] \supset$ $\left[x_{A}, x_{B}\right] \supseteq\left[x_{s 1}, x_{s 2}\right]$, the integral is to be considered as a Cauchy principal value, $\left(u_{\pi}, v_{\pi}\right)$ indicates the intersection of the PI-line on the detector, the constant term $P_{0}$ indicates the cone-beam projection of the object function along the PI-line, the backprojection image $g_{\pi}\left(x_{\pi}^{\prime}, \lambda_{1}, \lambda_{2}\right)$ on the PI-line segment is determined by

$$
g_{\pi}\left(x_{\pi}^{\prime}, \lambda_{1}, \lambda_{2}\right)=\left.\int_{\lambda_{1}}^{\lambda_{2}} \frac{d \lambda}{\left|\vec{r}^{\prime}-\vec{r}_{0}(\lambda)\right|} \frac{d}{d \lambda} P(u, v, \lambda)\right|_{\hat{\beta}} .
$$

For given $\left(x_{\pi}^{\prime}, \lambda_{1}, \lambda_{2}\right), \vec{r}^{\prime}$ is determined by (6) and $(u, v)$ are determined by

$$
u=\frac{S \vec{r}^{\prime} \cdot \hat{\mathrm{e}}_{u}(\lambda)}{R-\vec{r}^{\prime} \cdot \hat{\mathrm{e}}_{w}(\lambda)} \quad \text { and } \quad v=\frac{S \vec{r}^{\prime} \cdot \hat{\mathrm{e}}_{v}(\lambda)}{R-\vec{r}^{\prime} \cdot \hat{\mathrm{e}}_{w}(\lambda)} .
$$

It can be seen that the BPF algorithm reconstructs the image on a PI-line segment by first backprojecting the data derivatives onto the PI-line segment [i.e., the integration over $\lambda$ in (8)] and then performing a 1-D filtering along the PI-line. [i.e., the integration over $x_{\pi}^{\prime}$ in (7)].

\section{B. Circular Cone-Beam Scans and Virtual PI-Lines}

For circular cone-beam CT, the physical source trajectory is within a plane (i.e., the so-called midplane). Without loss of generality, the circular trajectory is assumed to be within the plane at $z=0$ and thus can be expressed as

$$
\vec{r}_{c}(\lambda)=(R \cos \lambda, R \sin \lambda, 0)^{T} .
$$

A PI-line intersecting two points on the trajectory can be defined meaningfully within the trajectory plane, i.e., the midplane. For an off-midplane (i.e., $z \neq 0$ ), however, no actual trajectory and, consequently, no actual PI-lines exist. Therefore, the BPF algorithm for helical cone-beam CT cannot be applied directly to reconstructing images in off-midplanes from circular cone-beam data.

In an attempt to derive an approximate BPF algorithm for circular cone-beam CT, we introduce the concept of virtual trajectory and virtual PI-line segments [29]. As illustrated in Fig. 2, a virtual circular trajectory within an off-midplane at $z \neq 0$ uses the $z$-axis as its central axis and has a radius $R$ identical to that of the actual circular trajectory in the plane at $z=0$. Thus, the collection of the actual and a stack of virtual circular trajectories within planes at different $z$ encloses a 3-D cylindrical volume, which is referred to as the virtual cylinder. We define a straight line segment connecting any two points on the actual circular trajectory at $z=0$ as an actual PI-line segment. Similarly, we define a straight line segment connecting any two points on a virtual circular trajectory at $z \neq 0$ as a virtual PI-line segment.

For a helical cone-beam scan, a point within the helix cylinder can be specified completely by three PI-line coordinates $\left(x_{\pi}, \lambda_{1}, \lambda_{2}\right)$ through (6), where $x_{\pi}$ indicates the location of the point on a PI-line segment specified by $\left(\lambda_{1}, \lambda_{2}\right)$. For the circular cone-beam scan, however, because the actual or virtual trajectory is within a plane, we need four PI-line parameters

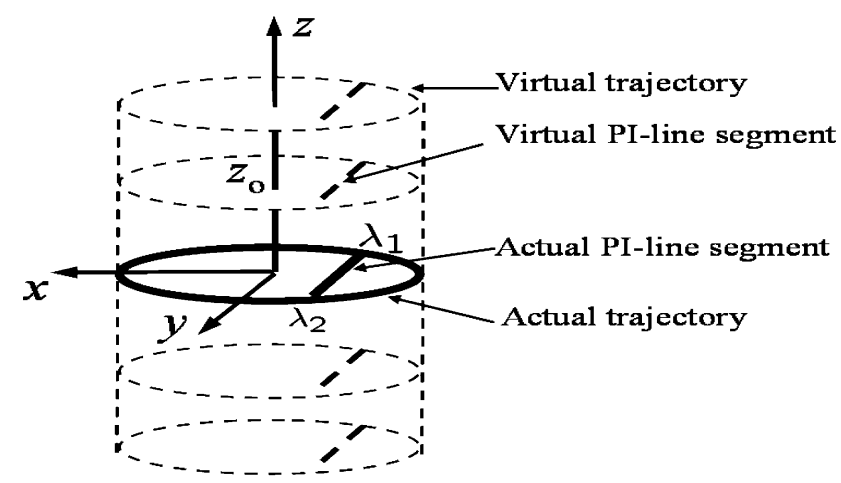

Fig. 2. Illustration of the actual and virtual circular trajectories, the actual and virtual PI-line segments.

$\left(x_{\pi}, \lambda_{1}, \lambda_{2}, z_{0}\right)$ to identify a point within the virtual cylinder. In addition to $x_{\pi}, \lambda_{1}$, and $\lambda_{2}$, the parameter $z_{0}$ is needed to indicate the plane that contains the actual or virtual trajectories. These PI-line coordinates are related to the fixed coordinates through

$$
\vec{r}=\vec{r}_{c 1}+x_{\pi} \hat{\mathrm{e}}_{\pi c}
$$

where

$$
\begin{aligned}
& \vec{r}_{c 1}=\frac{\vec{r}_{c}\left(\lambda_{2}\right)+\vec{r}_{c}\left(\lambda_{1}\right)}{2}+z_{0} \hat{\mathrm{e}}_{v} \\
& \hat{\mathrm{e}}_{\pi c}=\frac{\vec{r}_{c}\left(\lambda_{2}\right)-\vec{r}_{c}\left(\lambda_{1}\right)}{\left|\vec{r}_{c}\left(\lambda_{2}\right)-\vec{r}_{c}\left(\lambda_{1}\right)\right|} .
\end{aligned}
$$

\section{BPF Algorithm for Circular Cone-Beam CT}

For simplicity of discussion below, we will refer to an actual or a virtual PI-line segment as a PI-line segment. Let $x_{\pi 1}$ and $x_{\pi 2}$ denote the two end points of a PI-line segment. Also, we use $x_{s 1}$ and $x_{s 2}$ to denote the two end points of the intersection of a PI-line segment with the support of the object function; and we refer to this intersection as the support segment on the PI-line. Because the object function is enclosed completely within the virtual cylinder, we have $\left[x_{\pi 1}, x_{\pi 2}\right] \supset\left[x_{s 1}, x_{s 2}\right]$.

We present below a modified BPF algorithm for circular cone-beam CT to reconstruct exact images on actual PI-line segments and approximate images on virtual PI-line segments. Let $f_{\pi c}\left(x_{\pi}, \lambda_{1}, \lambda_{2}, z_{0}\right)$ denote the image on a virtual PI-line segment when $z_{0} \neq 0$ or the image on an actual PI-line segment when $z_{0}=0$. This modified BPF algorithm for circular cone-beam CT can be expressed as

$$
\begin{aligned}
f_{\pi c}\left(x_{\pi}, \lambda_{1}, \lambda_{2}, z_{0}\right) \\
=\frac{1}{2 \pi^{2}} \frac{1}{\sqrt{\left(x_{B}-x_{\pi}\right)\left(x_{\pi}-x_{A}\right)}} \\
\quad \times\left[\int_{x_{A}}^{x_{B}} \frac{d x_{\pi}^{\prime}}{x_{\pi}-x_{\pi}^{\prime}} \sqrt{\left(x_{B}-x_{\pi}^{\prime}\right)\left(x_{\pi}^{\prime}-x_{A}\right)}\right. \\
\left.\quad \times g_{\pi c}\left(x_{\pi}^{\prime}, \lambda_{1}, \lambda_{2}, z_{0}\right)+2 \pi P_{c 0}\right]
\end{aligned}
$$

where $x_{A}$ and $x_{B}$ are any points satisfying $\left[x_{\pi 1}, x_{\pi 2}\right] \supset$ $\left[x_{A}, x_{B}\right] \supseteq\left[x_{s 1}, x_{s 2}\right]$, the integral is to be considered as 
a Cauchy principal value, the backprojection image on the PI-line segment can be expressed as [19], [27]

$$
g_{\pi c}\left(x_{\pi}^{\prime}, \lambda_{1}, \lambda_{2}, z_{0}\right)=\left.\int_{\lambda_{1}}^{\lambda_{2}} \frac{d \lambda}{\left|\vec{r}^{\prime}-\vec{r}_{0}(\lambda)\right|} \frac{d}{d \lambda} P(u, v, \lambda)\right|_{\hat{\beta}}
$$

and the constant term $P_{c 0}$ indicates the line integral of the object function along the PI-line. Notice that $P_{c 0}$ cannot be obtained from the data function $P(u, v, \lambda)$ when the PI-line is in an offmidplane $(z \neq 0)$. Therefore, we use

$$
P_{c 0}=\frac{P\left(u_{1}, v_{1}, \lambda_{1}\right)+P\left(u_{2}, v_{2}, \lambda_{2}\right)}{2}
$$

to approximate the line integral of the object function along the PI-line, where $P\left(u_{1}, v_{1}, \lambda_{1}\right)$ and $P\left(u_{2}, v_{2}, \lambda_{2}\right)$ indicate the cone-beam projection of the point $\left.\left(\left(x_{\pi 1}+x_{\pi 2}\right) / 2\right), \lambda_{1}, \lambda_{2}, z_{0}\right)$, i.e., the central point on the PI-line segment, from view angle $\lambda_{1}$ and $\lambda_{2}$, respectively. In (13) and (14), for given $\left(x_{\pi}^{\prime}, \lambda_{1}, \lambda_{2}, z_{0}\right)$, $\vec{r}^{\prime}$ is obtained by use of (11), whereas $(u, v)$ are determined through

$$
u=\frac{S \vec{r}^{\prime} \cdot \hat{\mathrm{e}}_{u}(\lambda)}{R-\vec{r}^{\prime} \cdot \hat{\mathrm{e}}_{w}(\lambda)} \quad \text { and } \quad v=\frac{S z_{0}}{R-\vec{r}^{\prime} \cdot \hat{\mathrm{e}}_{w}(\lambda)} .
$$

Comparison of (7) and (13) indicates that the BPF algorithm for circular cone-beam CT and helical cone-beam CT are formally identical. However, the constant terms and backprojection images in the two algorithms are different.

Furthermore, using the approach similar to those in [19], [27], we can re-express the backprojection image in (14) as

$$
\begin{aligned}
& g_{\pi c}\left(x_{\pi}^{\prime}, \lambda_{1}, \lambda_{2}, z_{0}\right)=\int_{\lambda_{1}}^{\lambda_{2}} d \lambda \frac{S^{2}}{\left[R-\vec{r}^{\prime} \cdot \hat{\mathrm{e}}_{w}(\lambda)\right]^{2}} \\
& \times \frac{\partial}{\partial u}\left[\frac{R}{A} P(u, v, \lambda)\right]+\left.\frac{P(u, v, \lambda)}{\left|\vec{r}^{\prime}-\vec{r}_{c}(\lambda)\right|}\right|_{\lambda_{1}} ^{\lambda_{2}}
\end{aligned}
$$

where

$$
A=\sqrt{u^{2}+v^{2}+S^{2}} .
$$

In practical situations, the discrete samples on the detector are generally denser than those over a scanning trajectory. Therefore, (17) generally yields numerically more accurate backprojection image than does (14).

\section{Weighted BPF Algorithm for Exploitation of Data Redundancy}

As described before, the BPF algorithm reconstructs the image on a PI-line segment specified by $\lambda_{1}$ and $\lambda_{2}$ by use of data acquired over an angular range $\left[\lambda_{1}, \lambda_{2}\right]$. Let $\left[\lambda_{\min }, \lambda_{\max }\right]$ denote the total scanning angular range. Obviously, a PI-line segment satisfies $\left[\lambda_{1}, \lambda_{2}\right] \subseteq\left[\lambda_{\min }, \lambda_{\max }\right]$ is reconstructible. Therefore, as shown in Fig. 3, data acquired over the angular ranges $\left[\lambda_{\min }, \lambda_{1}\right)$ and $\left(\lambda_{2}, \lambda_{\max }\right]$ constitute redundant information for image reconstruction on an actual PI-line segment specified by $\lambda_{1}$ and $\lambda_{2}$ in the midplane. On the other hand, for a virtual PI-line specified by $\lambda_{1}$ and $\lambda_{2}$ in the off-midplane, the

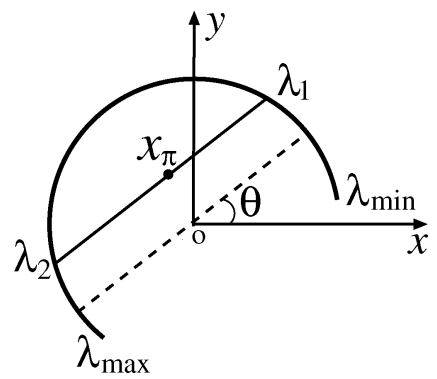

Fig. 3. Illustration of the data redundancy. Actual scanning angular range is $\left[\lambda_{\min }, \lambda_{\max }\right]$. PI-line segment to be reconstructed is specified by $\left(\lambda_{1}, \lambda_{2}\right)$, where $\left[\lambda_{1}, \lambda_{2}\right] \in\left[\lambda_{\min }, \lambda_{\max }\right]$. Obviously, data acquired over the angular ranges $\left[\lambda_{\min }, \lambda_{1}\right)$ and $\left(\lambda_{2}, \lambda_{\max }\right]$ contain redundant information with respect to the image reconstruction on the PI-line segment specified by $\lambda_{1}$ and $\lambda_{2}$. $x_{\pi}$ represents an arbitrary point on the PI-segment, and $\theta$ denotes the angle between the PI-line segment and the $x$ axis.

angular ranges $\left[\lambda_{\min }, \lambda_{1}\right)$ and $\left(\lambda_{2}, \lambda_{\max }\right]$ on the corresponding virtual trajectory do not generally contain truly redundant data information because no rays in the cone beam are within that off-midplane. However, data in the angular ranges $\left[\lambda_{\min }, \lambda_{1}\right)$ and $\left(\lambda_{2}, \lambda_{\max }\right]$ on a virtual trajectory can be treated as approximate redundant information. We present below a weighted $\mathrm{BPF}$ algorithm that exploits the truly redundant information for actual PI-line segments in the midplane and the approximate redundant information for virtual PI-line segments in the off-midplanes.

Following the strategy for exploiting data redundancy in fanbeam CT [24], we have derived a weighted BPF algorithm for exploiting data redundancy in circular cone-beam CT, which has a form identical to that of (13) except that the backprojection image should be replaced by

$$
\begin{aligned}
& g_{\pi c}^{(\omega)}\left(x_{\pi}^{\prime}, \lambda_{1}, \lambda_{2}, z_{0}\right)=\int_{\lambda_{\min }}^{\lambda_{\max }} d \lambda \frac{S^{2}}{\left[R-\vec{r}^{\prime} \cdot \hat{\mathrm{e}}_{w}(\lambda)\right]^{2}} \\
& \times \frac{\partial}{\partial u}\left[\frac{R}{A} \omega(u, \lambda) P(u, v, \lambda)\right]+\left.\frac{\omega(u, \lambda) P(u, v, \lambda)}{\left|\vec{r}^{\prime}-\vec{r}_{c}(\lambda)\right|}\right|_{\lambda_{\min }} ^{\lambda_{\max }}
\end{aligned}
$$

where the weighting function $\omega(u, \lambda)$ satisfies

$$
\omega(u, \lambda)-\omega\left(-u, \lambda+\pi-2 \arctan \frac{u}{S}\right)=1 .
$$

Inspection of (20) shows that $\omega(u, \lambda)$ is discontinuous in $u$ and $\lambda$ and thus prevents an accurate and stable numerical implementation of (19). In an attempt to avoid such a discontinuity in numerical implementation, without loss of generality, we assume that, as shown in Fig. 3, a PI-line segment has an angle $\theta$ relative to the $x$ axis and that $\omega(u, \lambda)=H_{0}(u, \lambda) \omega_{0}(u, \lambda)$, where $\omega_{0}(u, \lambda)$ is a function satisfying [30], [31]

$$
\omega_{0}(u, \lambda)+\omega_{0}\left(-u, \lambda+\pi-2 \arctan \frac{u}{S}\right)=1
$$

and function $H_{0}(u, \lambda)$ is given by

$$
H_{0}(u, \lambda)=\left\{\begin{array}{ll}
1 & \left(\lambda-\arctan \frac{u}{S}\right) \in(\theta, \theta+\pi) \\
-1 & \left(\lambda-\arctan \frac{u}{S}\right) \notin(\theta, \theta+\pi)
\end{array} .\right.
$$

One can readily demonstrate that $\omega_{0}(u, \lambda) H_{0}(u, \lambda)$ satisfies (20). In Appendix, following the similar strategy in [22], we 
show that the weighted backprojection image in (19) can be rewritten as

$$
\begin{aligned}
g_{\pi c}^{(\omega)} & \left(x_{\pi}^{\prime}, \lambda_{1}, \lambda_{2}, z_{0}\right) \\
= & \int_{\lambda_{\min }}^{\lambda_{\max }} d \lambda \frac{S^{2}}{\left[R-\vec{r}^{\prime} \cdot \hat{\mathrm{e}}_{w}(\lambda)\right]^{2}} H_{0}(u, \lambda) \\
& \times \frac{\partial}{\partial u}\left[\frac{R}{A} \omega_{0}(u, \lambda) P(u, v, \lambda)\right] \\
& +\left.\frac{H_{0}(u, \lambda) \omega_{0}(u, \lambda) P(u, v, \lambda)}{\left|\vec{r}^{\prime}-\vec{r}_{c}(\lambda)\right|}\right|_{\lambda_{\min }} ^{\lambda_{\max }} \\
& +\left.\frac{2 \omega_{0}(u, \lambda) P(u, v, \lambda)}{\left|\vec{r}^{\prime}-\vec{r}_{c}(\lambda)\right|}\right|_{\lambda_{1}} ^{\lambda_{2}} .
\end{aligned}
$$

It can be seen in (23) that, as long as $\omega_{0}(u, \lambda)$ is smooth, it is possible to compute accurately the derivative in the first term in (23). The result in (23) includes two boundary terms determined by the actual scanning angular range specified by $\lambda_{\min }$ and $\lambda_{\max }$ and by the angular range determined by $\lambda_{1}$ and $\lambda_{2}$ of a PI-line segment. For a full scan $\lambda_{\min }=0, \lambda_{\max }=2 \pi$, the first boundary term vanishes, and only the second boundary term remains. Noo et al. obtained a similar result for full-scan fan-beam case in [22, eq. (35)]. A smooth weighting function $\omega_{0}(u, \lambda)$ can readily be constructed in the case of a full-scan, a short-scan [30], [31] or any scan with angular range between a short-scan and a full-scan [30]. However, when the scanning angular range is less than a short-scan, the construction of a smooth weighting function is not obvious.

\section{E. ROI-Image Reconstruction in Circular Cone-Beam CT}

A unique property of the proposed BPF algorithms is that they can, from circular cone-beam data containing transverse truncations, reconstruct ROI images with an accuracy comparable to that obtained from nontruncated circular cone-beam data.

For a PI-line segment specified by $\lambda_{1}$ and $\lambda_{2}$, as shown in (13), one needs knowledge of the backprojection only in $\left[x_{A}, x_{B}\right]$ for exact image reconstruction. Such knowledge can be obtained from data on the cone-beam projections of $\left[x_{A}, x_{B}\right]$ for $\lambda \in\left[-\lambda_{1}, \lambda_{2}\right]$ [see (17)] or for $\lambda \in\left[\lambda_{\min }, \lambda_{\max }\right]$ (see (23)). Because $\left[x_{s 1}, x_{s 2}\right] \subseteq\left[x_{A}, x_{B}\right]$, one in effect needs data only on the cone-beam projections of the support segment. Therefore, as long as the support segment is always illuminated fully at $\lambda \in\left[\lambda_{1}, \lambda_{2}\right]$ or at $\lambda \in\left[\lambda_{\min }, \lambda_{\max }\right]$, sufficient data can be collected for computation of the backprojection image, even if the illumination at these views may not completely cover the entire object function (i.e., the projections are truncated). Therefore, an image on the PI-line segment free of data-truncation artifacts can be reconstructed.

Now, consider a set of PI-line segments that completely fill in an ROI. Without loss of generality, we assume that the maximum angular range required by these PI-line segments is $\left[\lambda_{\min }, \lambda_{\max }\right]$. If the support segments on these PI-line segments are fully illuminated for $\lambda \in\left[\lambda_{\min }, \lambda_{\max }\right]$, based upon the observation above, even if the FOV of the illumination is smaller than the actual size of the entire object support, (i.e., the projections are truncated), one can reconstruct images on these PI-line segments and consequently the image within the ROI that are free of the impact of data truncation.

\section{NUMERICAL STUDIES}

We have performed quantitative studies to validate and evaluate the proposed algorithms in Sections II-C and II-D by using both computer-simulation data and real-experimental data.

\section{A. Quantitative Studies With Computer-Simulation Data}

In the computer-simulation studies, we have considered a circular cone-beam configuration in which the trajectory has a radius of $290 \mathrm{~mm}$ and a source-detector distance of $450 \mathrm{~mm}$. A modified, low-contrast 3-D Shepp-Logan phantom was used, which has an ellipsoidal support with radii of 49,98 , and $90 \mathrm{~mm}$ along $x, y$, and $z$ axis, respectively. As compared to the standard 3-D Shepp-Logan phantom, this phantom has a shorter support along $x$ axis and a longer support along $y$ axis for demonstrating the transverse truncation effect. The detector plane consists of $256 \times 256$ elements each of which has an area of $1.3 \times 1.3 \mathrm{~mm}^{2}$. We have generated nontruncated circular cone-beam data at 300 projection views uniformly distributed over $2 \pi$; and the transversely truncated data were obtained by setting the values in 35 bins on each side of the detector panel to zero. We have also generated noisy data by adding Gaussian noise to the noiseless data. In an attempt to show the low-contrast structures, the standard deviation of the Gaussian noise was selected to be about $0.063 \%$ of the maximum value of noiseless data. The proposed BPF algorithms for circular cone-beam CT were applied to these full and truncated data to reconstruct full and ROI images. To further compare the BPF algorithms with the FDK algorithm when there is no truncation, we generated another circular cone-beam dataset with a Defrise disk phantom. The radius of the circular trajectory is $576 \mathrm{~mm}$. The source-detected distance is $736 \mathrm{~mm}$. The cone-angle is $20^{\circ}$ with an FOV size of $200 \mathrm{~mm}$.

1) Image Reconstruction From Nontruncated Data: We display in Fig. 4 images within two-dimensional (2-D) slices in 3-D images reconstructed by use of the FDK algorithm (upper row), the BPF algorithm in (17) (middle row), and the weighted BPF algorithm in (19) (lower row), respectively, from nontruncated, noiseless cone-beam data generated with the Shepp-Logan phantom. The images from the weighted BPF algorithm were obtained using the complete $2 \pi$ range of data with a weight of $1 / 2$. No smoothing filter was applied in all the reconstruction. The first to fourth columns represent images within planes at $x=0, y=25 \mathrm{~mm}, z=0$, $z=6.4 \mathrm{~mm}$, respectively. The display window is [1.0, 1.04]. We also show in Fig. 5 image profiles obtained by use of the weighted BPF algorithm in (19) (solid curve) and the FDK algorithm (dashed-dotted curve) on lines specified by (a) $x=0$, $y=25 \mathrm{~mm}$ and (b) $x=17 \mathrm{~mm}, z=0$, respectively. For a comparison, we have also plotted the true profiles (dotted curves) in Fig. 5.

We show in Fig. 6 images within 2-D slices in 3-D images reconstructed by use of the FDK algorithm (upper row), the BPF algorithm in (17) (middle row), and the weighted BPF algorithm in (19) (lower row), respectively, from nontruncated, noisy cone-beam data generated with the Shepp-Logan phantom. Again, the first to fourth columns represent images 


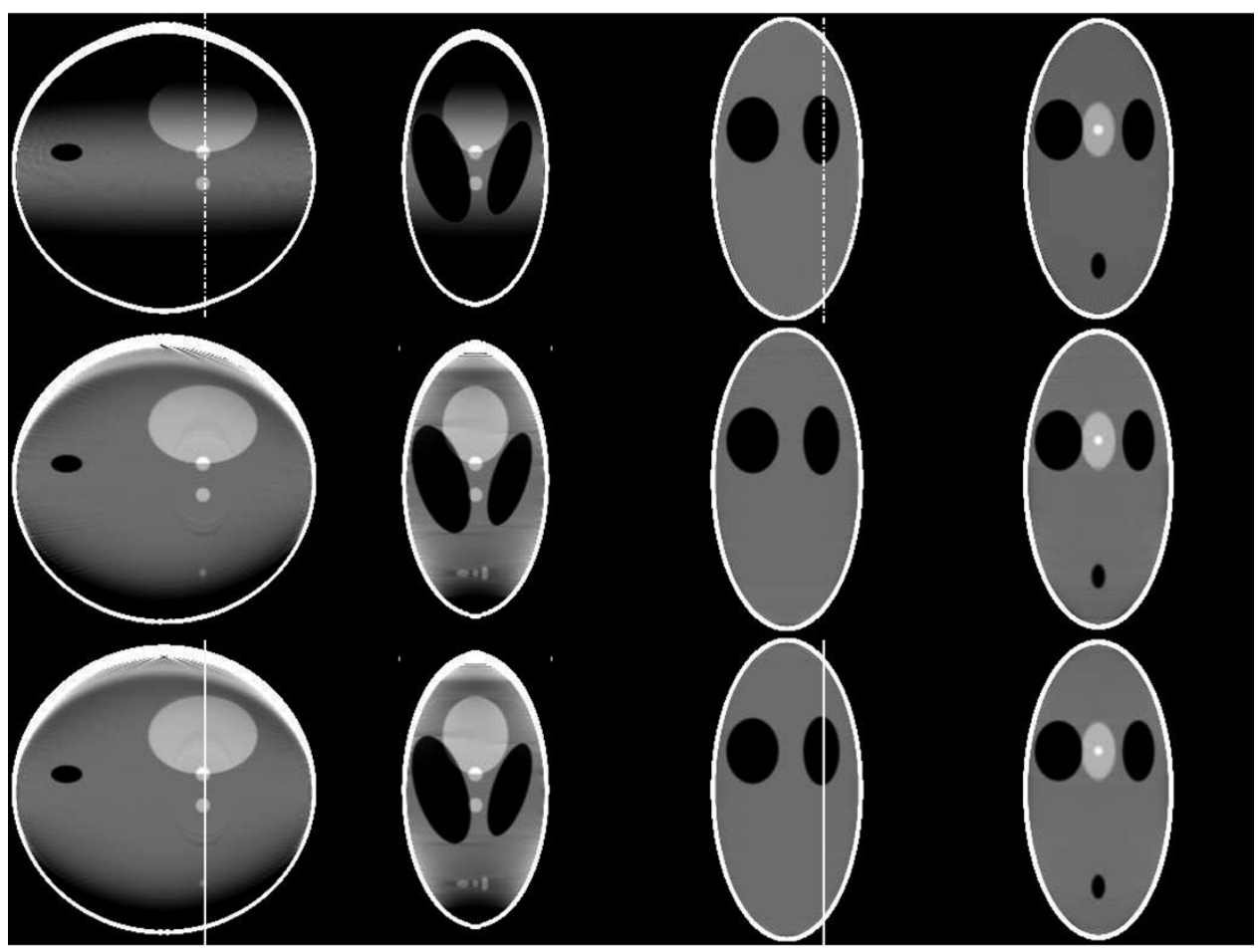

Fig. 4. Two-dimensional slices in 3-D images reconstructed by use of the FDK algorithm (upper row), the BPF algorithm in (17) (middle row) and the weighted BPF algorithm in (19) (lower row), respectively, from noiseless data containing no truncation. First to fourth columns represent the 2-D slices in planes of $x=0$, $y=25 \mathrm{~mm}, z=0, z=6.4 \mathrm{~mm}$, respectively. Display window is [1.0, 1.04].

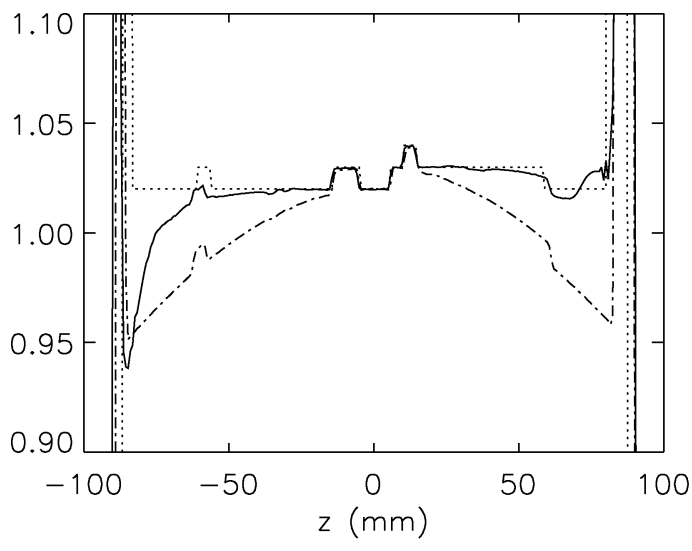

(a)

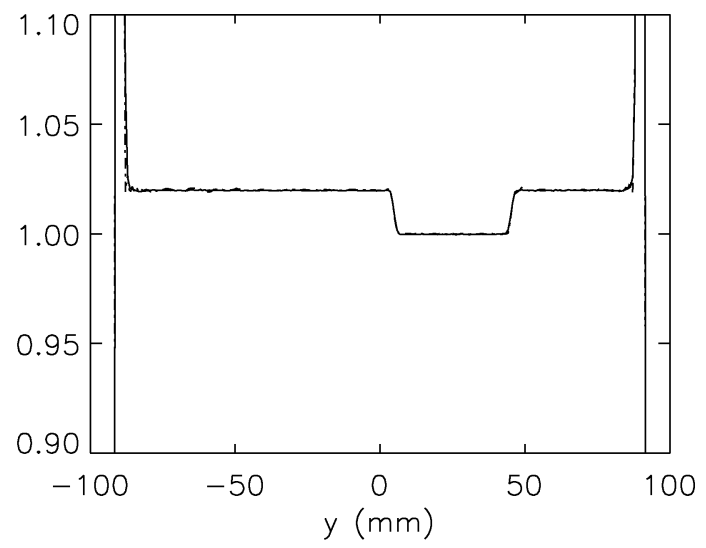

(b)

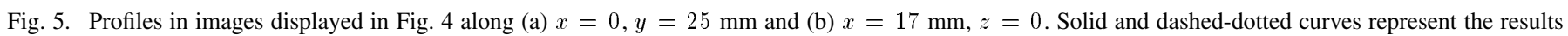
obtained by use of the weighted BPF algorithm in (19) and the FDK algorithm, respectively. True profiles (dotted curve) in the original phantom are also plotted.

within planes at $x=0, y=25 \mathrm{~mm}, z=0, z=6.4 \mathrm{~mm}$, respectively. The display window is $[1.0,1.04]$. These results suggest that the weighted BPF algorithm can exploit redundant information within the data for suppression of image noise.

Fig. 7 compares the reconstruction results from nontruncated data generated with the disk phantom. Sagittal slices $(x=0)$ in the 3-D images reconstructed by use of the FDK algorithm, the BPF algorithm in (17), and the weighted BPF algorithm in (19) are displayed in Fig. 7(a)-(c), respectively. The display window is $[0,2]$. The profiles along central columns in image Fig. 7 (a) and Fig. 7(c) are plotted in Fig. 7(d) with dashed-dotted and solid curves, respectively. The corresponding true profile in the original disk phantom is also plotted with a dotted curve. It can be observed that the BPF algorithms perform differently from the FDK algorithm in terms of shape distortion and intensity drop in the off-midplane.

2) ROI-Image Reconstruction From Truncated Data: We have applied the proposed BPF algorithms to reconstructing ROI images from the computer simulated data containing truncations. In Fig. 8, we display ROI images reconstructed by using the FDK algorithm (upper row), the BPF algorithm in (17) (middle row), and the weighted BPF algorithm in (19) (lower row), respectively, from truncated, noiseless cone-beam data. The first to fourth columns represent images within planes at $x=0, y=25 \mathrm{~mm}, z=0, z=6.4 \mathrm{~mm}$, respectively. The display window is $[1.0,1.04]$. We also show in Fig. 9 


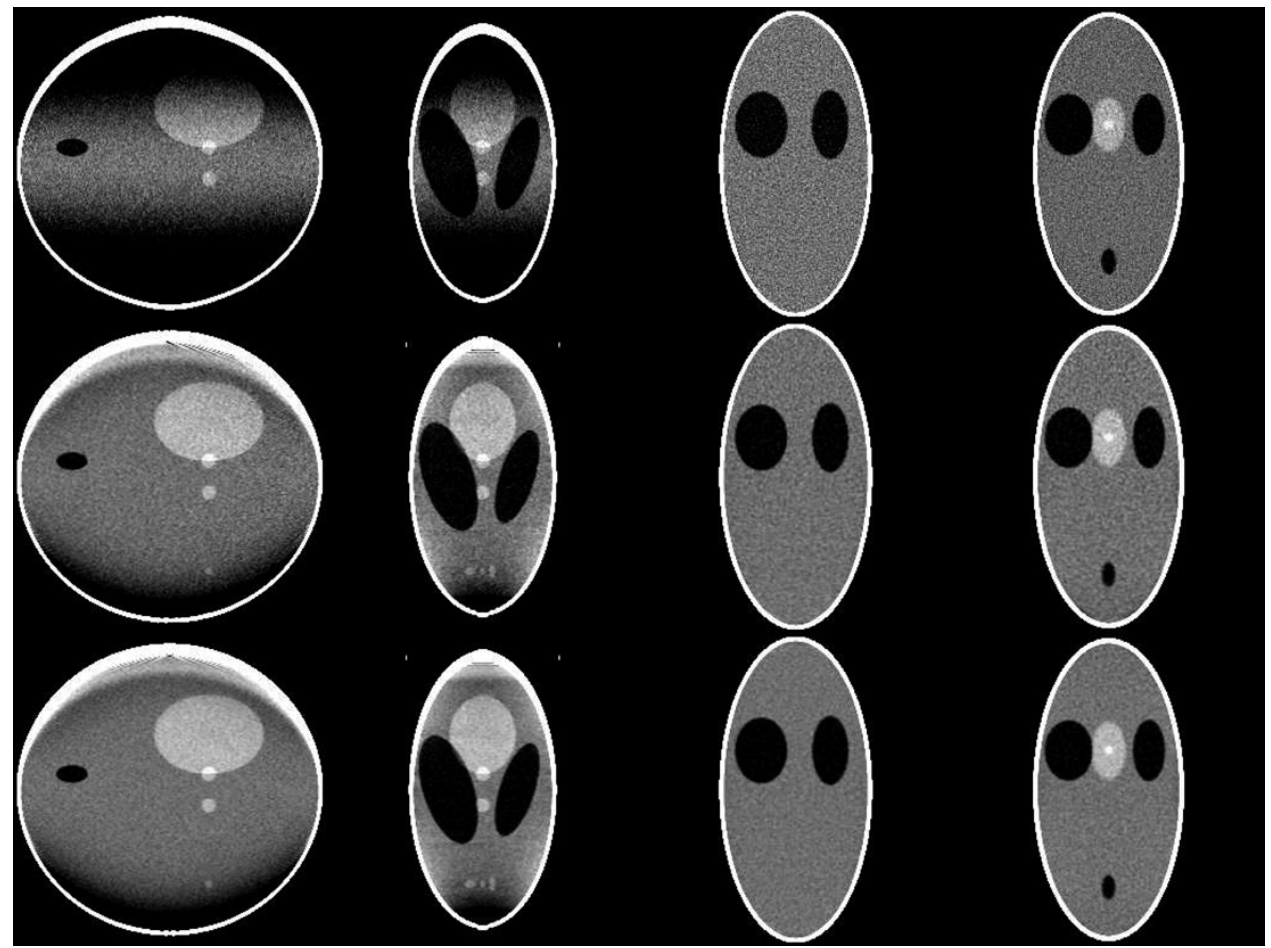

Fig. 6. Two-dimensional slices in 3-D images reconstructed by use of the FDK algorithm (upper row), the BPF algorithm in (17) (middle row) and the weighted $\mathrm{BPF}$ algorithm in (19) (lower row), respectively, from noisy data containing no truncation. First to fourth columns represent the 2-D slices in planes of $x=0$, $y=25 \mathrm{~mm}, z=0, z=6.4 \mathrm{~mm}$, respectively. Display window is $[1.0,1.04]$.

image profiles obtained by use of the weighted BPF algorithm in (19) (solid curve) and the FDK algorithm (dashed-dotted curve) on lines specified by $x=0, y=25 \mathrm{~mm}$ [Fig. 9(a)] and $x=17 \mathrm{~mm}, z=0$ [Fig. 9(b)], respectively. For a comparison, we have also plotted the true profiles (dotted curve) in Fig. 9. From these results, one can observe that data truncation can result in severe artifacts in images obtained with the FDK algorithm. However, ROI images reconstructed by use of the proposed BPF algorithms are free of truncation artifacts and are in fact identical to the images within the same ROI's reconstructed by use of nontruncated data.

We also show in Fig. 10 ROI images reconstructed by using the FDK algorithm (upper row), the BPF algorithm in (17) (middle row), and the weighted BPF algorithm in (19) (lower row), respectively, from truncated, noisy cone-beam data. The first to fourth columns represent images within planes at $x=0$, $y=25 \mathrm{~mm}, z=0, z=6.4 \mathrm{~mm}$, respectively. The display window is [1.0, 1.04]. Again, it can be observed that data truncation can result in severe artifacts in images obtained with the FDK algorithm. However, ROI images reconstructed by use of the proposed BPF algorithms are free of truncation artifacts. The results also suggest that the weighted BPF algorithm can exploit redundant information within data for suppression of image noise.

\section{B. Quantitative Studies With Experimental Data}

We have performed evaluation studies of the proposed algorithms by using real data acquired with a simulator CT (Acuity, Varian Medical Systems) from a head phantom.
1) Experimental Acquisition of Circular Cone-Beam Data: The simulator cone-beam CT system for radiation therapy consists of a kilovolt X-ray source, a patient couch, and an amorphous silicon flat-panel detector (Varian PaxScan 4030CB). It was operated for our measurements at a source to isocenter distance of $996.3 \mathrm{~mm}$ and a source to detector distance of 1490.8 $\mathrm{mm}$. The gantry rotates in an angular range of $\pm 185^{\circ}$ and with isocenter accuracy less than $1 \mathrm{~mm}$ diameter when there is no accessory load. The rotation speed is up to $360^{\circ} / \mathrm{min}$. The detector has $2048 \times 1536$ pixels, each with a size of $0.194 \times 0.194 \mathrm{~mm}^{2}$. In our measurement, the detector was operated in a $2 \times 2$ rebinned mode and the data were stored as 16-bit values. The fanand cone-angle are $15.2^{\circ}$ and $11.4^{\circ}$, respectively, where the fanangle is the angle in the midplane and cone-angle is the angle in the plane vertical to the midplane. Circular cone-beam data were collected from the head phantom at 683 projection views distributed over $2 \pi$. In the original dataset, the head phantom was almost covered by the total FOV and truncated only in a very small peripheral region. In an effort to stimulate a situation of data truncation, we manually set 227 columns of pixels in each side of the detector to zero.

2) Image Reconstruction From Real-Experimental Data: We have used both the original experimental data and the manually generated truncated data to reconstruct images. In Fig. 11, we show images reconstructed from the original data by use of the FDK algorithm (upper row), the BPF algorithm in (17) (middle row), and the weighted BPF algorithm in (19) (lower row), respectively. The first to fourth columns show the images within planes specified by $x=0, y=0, z=0$, and $z=-33.8 \mathrm{~mm}$. The display window is $[0.017,0.030] \mathrm{mm}^{-1}$. 


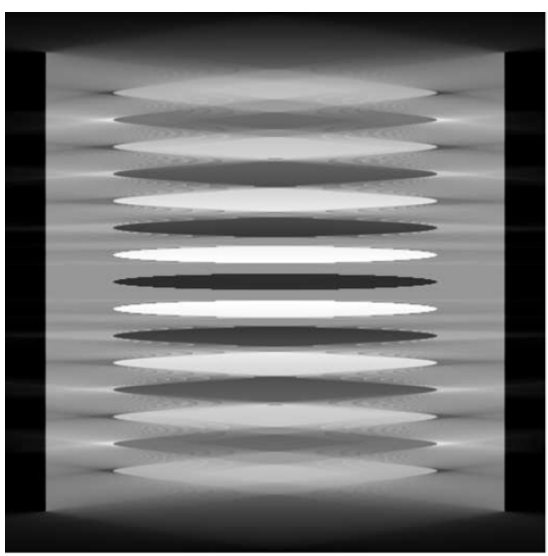

(a)

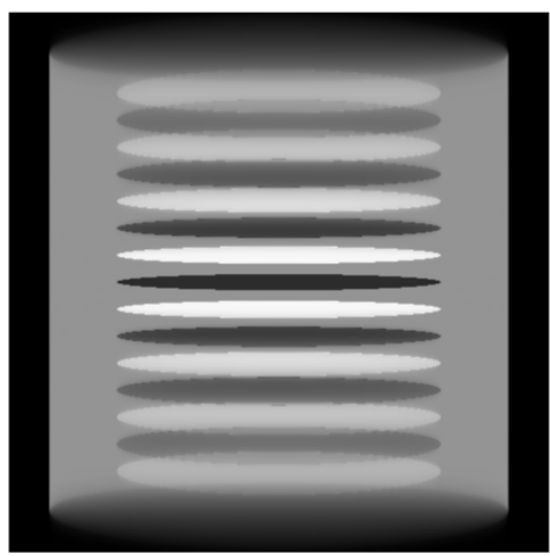

(c)

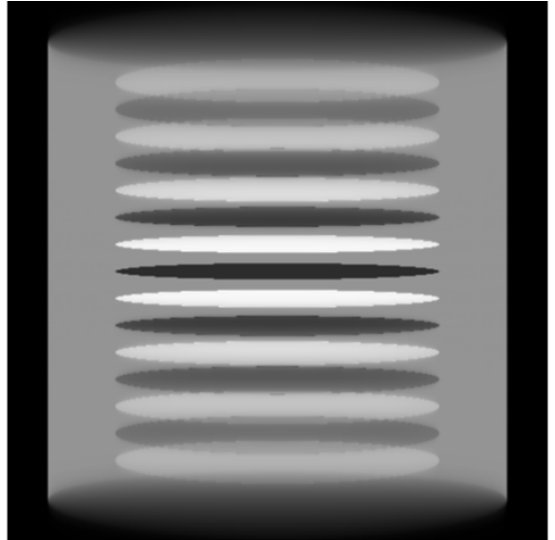

(b)

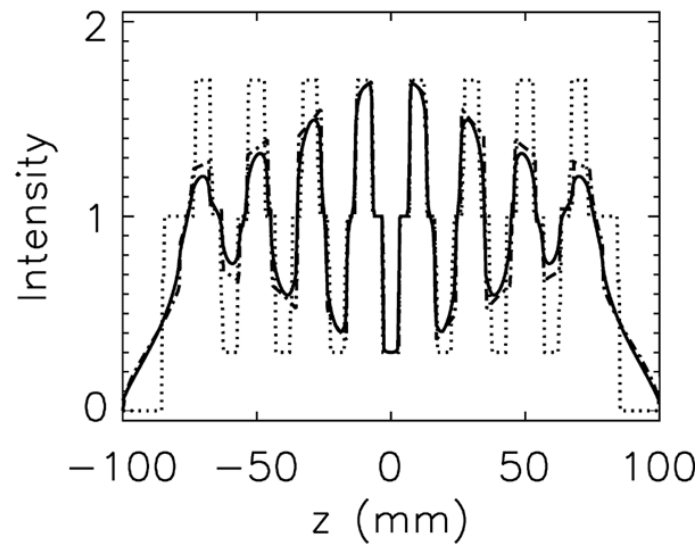

(d)

Fig. 7. Sagittal slices $(x=0)$ in 3-D images reconstructed by use of (a) the FDK algorithm, (b) the BPF algorithm in (17), and (c) the weighted BPF algorithm in (19), respectively, from noiseless data containing no truncation generated with a disk phantom. Display window is [0, 2]. Profiles along central columns in image (a) and (c) are plotted in (d) with dashed-dotted and solid curves, respectively. Corresponding true profile in the original phantom is also plotted with a dotted curve.

The displayed FOV size is $251.0 \times 251.0 \times 125.5 \mathrm{~mm}^{3}$. We also show in Fig. 12 profiles on lines specified by (a) $z=0$, $x=0$ and (b) $z=-33.8 \mathrm{~mm}, x=0$ in images displayed in Fig. 11 obtained by using the FDK algorithm (dotted curve) and the weighted BPF algorithm (solid curve). As can be seen from these results, in the situation of no truncation (almost), three algorithms generate similar images except that their noise properties are different. The images obtained with the FDK algorithm appear noisier than those obtained with our proposed two algorithms, but the resolution was not exactly matched either. Furthermore, the weighted BPF algorithm generates images with lower noise level and less artifacts than does the BPF algorithm because of the exploitation of the redundant information.

In Fig. 13, we show images reconstructed from the generated truncated data by use of the FDK algorithm (upper row), the BPF algorithm in (17) (middle row), and the weighted BPF algorithm in (19) (lower row), respectively. The first to fourth columns show the images within planes specified by $x=0$, $y=0, z=0$, and $z=-33.8 \mathrm{~mm}$. The display window is $[0.017,0.030] \mathrm{mm}^{-1}$. The displayed FOV size is $147.4 \times$ $147.4 \times 73.7 \mathrm{~mm}^{3}$. We also show in Fig. 14 profiles on lines specified by $z=0, x=0$ [Fig. 14(a)] and $z=-33.8 \mathrm{~mm}$, $x=0$ [Fig. 14(b)] in images displayed in Fig. 11 obtained by using the FDK algorithm (dotted curve) and the weighted BPF algorithm (solid curve).

Images displayed in Fig. 11 were obtained from data with almost no truncation, they can thus be considered as true images. Comparing results in Figs. 13 and 14 with those in Figs. 11 and 12 , one can observe that data truncation results in severe artifacts in images obtained with the FDK algorithm. In contrast, ROI images reconstructed by use of the proposed BPF algorithms are free of truncation artifacts.

\section{DISCUSSION}

In this work, we have presented new algorithms for image reconstruction from projection data acquired in circular cone-beam CT. These algorithms make use of the PI-line concept developed for exact image reconstruction in helical cone-beam CT. Image reconstruction on PI-lines allows for exact ROI image reconstruction from truncated data in the setting of a helical scan [19], [20]. In this article, we use the virtual PI-line concept that allows for approximate image reconstruction for the imaging volume away from the midplane of the circular-scan orbit. The most important feature of the new algorithms is that they allow for ROI reconstruction from 


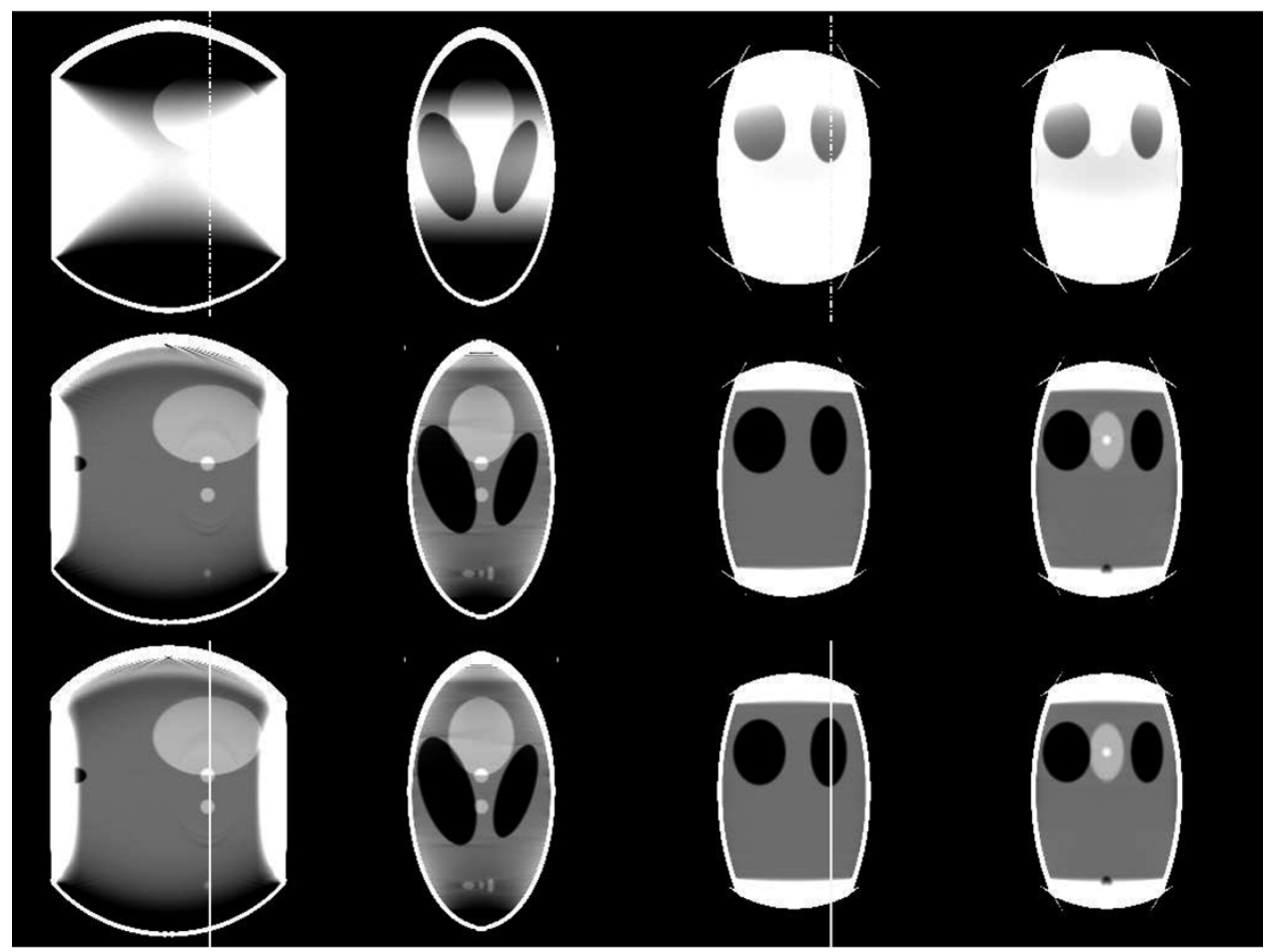

Fig. 8. Two-dimensional slices in 3-D images reconstructed by use of the FDK algorithm (upper row), the BPF algorithm in (17) (middle row) and the weighted BPF algorithm in (19) (lower row), respectively, from noiseless data generated from the truncated cone-beam scan. First to fourth columns represent the 2-D slices in planes of $x=0, y=25 \mathrm{~mm}, z=0, z=6.4 \mathrm{~mm}$, respectively. Display window is [1.0, 1.04].

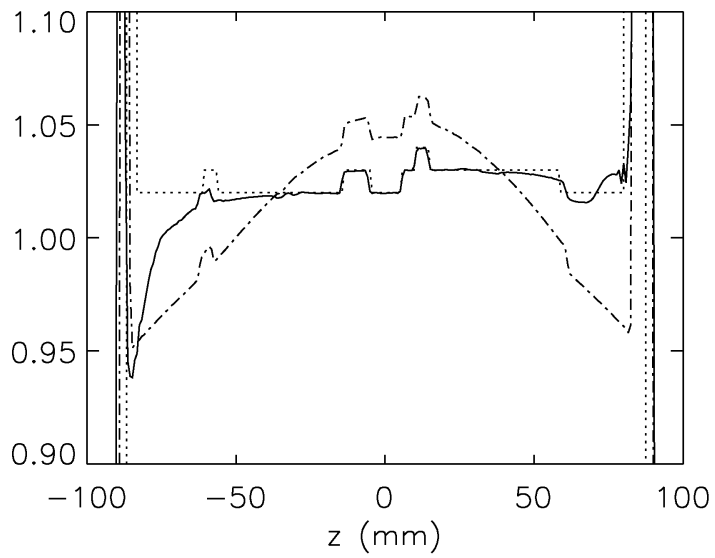

(a)

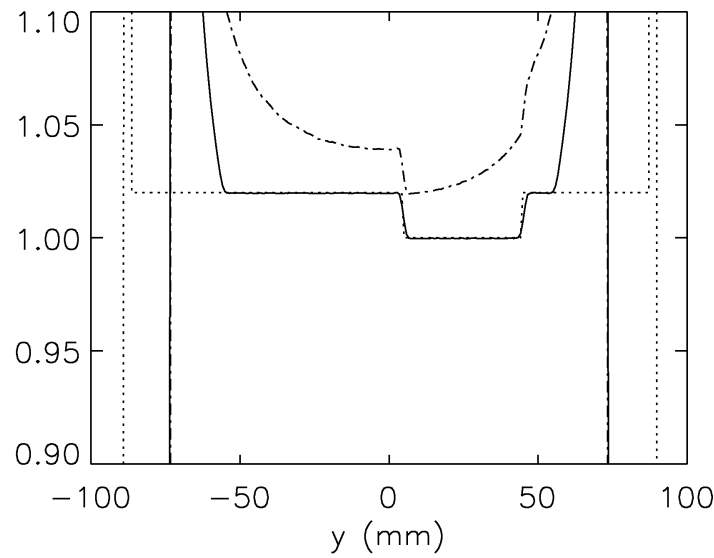

(b)

Fig. 9. Profiles in images displayed in Fig. 8 along (a) $x=0, y=25 \mathrm{~mm}$ and (b) $x=17 \mathrm{~mm}, z=0$. Solid and dashed-dotted curves represent the results obtained by use of our algorithm in (19) and the FDK algorithm, respectively. Ttrue profiles (dotted curve) in the original phantom are also plotted.

truncated data, free of the artifacts due to this truncation in the projection data.

Both the FDK and BPF algorithms can approximately reconstruct images from circular cone-beam data. The approximations invoked in the two algorithms are different, yielding different images within off-midplanes. In the absence of data truncation, the differences between images obtained with the two algorithms appear to be dependent on several factors such as cone angle, trajectory radius, and object structures. It will be interesting to analyze in future studies how the impact of approximations made in the two algorithms precisely differ from each other.
In the work reported here, we have used the virtual circular trajectories within planes parallel to the midplane to construct the virtual PI-lines. One can certainly choose other types of virtual trajectories and the corresponding virtual chords. Because the BPF algorithm for reconstructing chords of a general trajectory has been developed [32], one can readily apply it to reconstruct images on virtual chords. It is an interesting topic to investigate image reconstruction for different types of virtual trajectories and virtual chords. In particular, it is worthy to identify the virtual trajectory and conditions under which the identified trajectory performs more optimally than other possible virtual trajectories. 


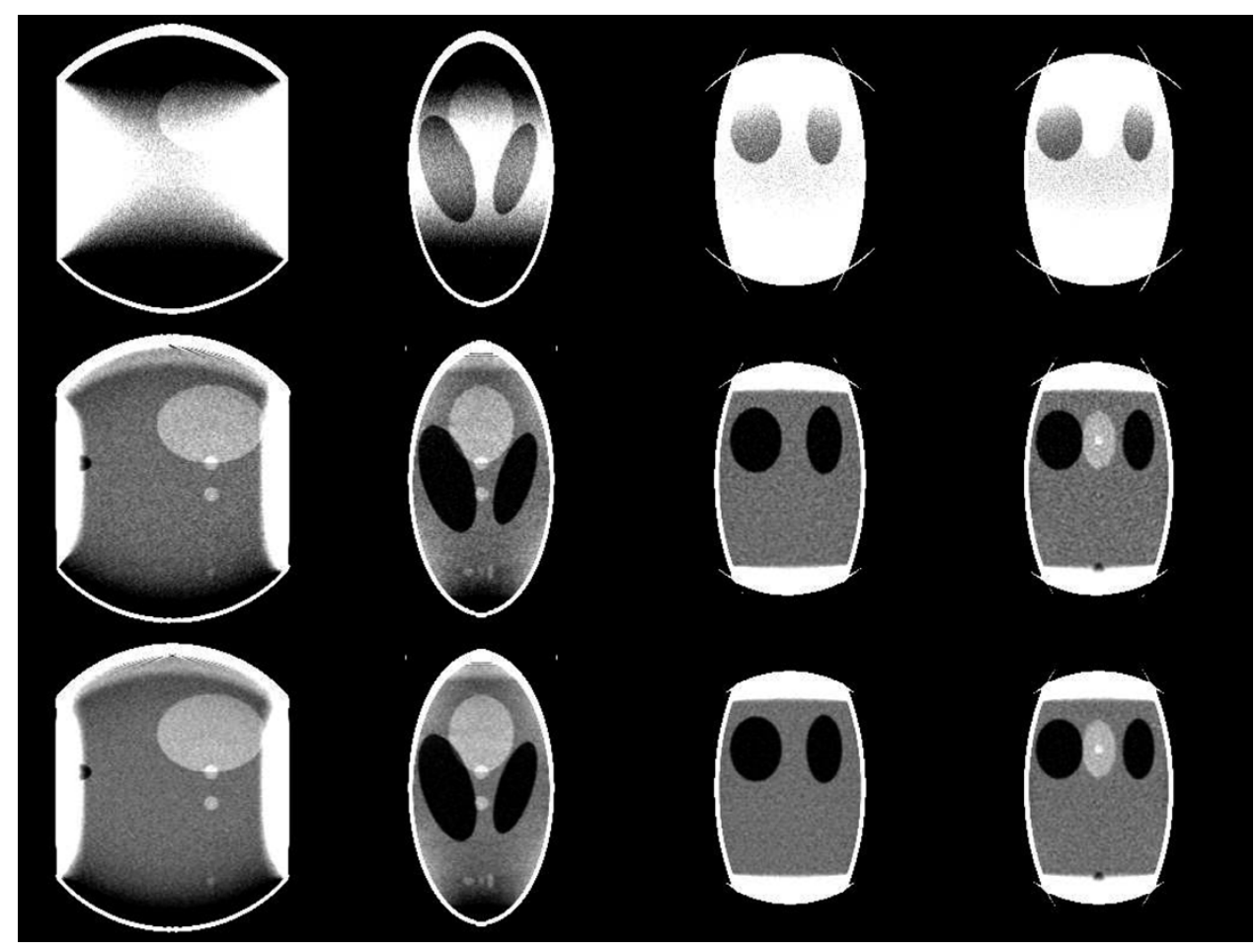

Fig. 10. Two-dimensional slices in 3-D images reconstructed by use of the FDK algorithm (upper row), the BPF algorithm in (17) (middle row), and the weighted BPF algorithm in (19) (lower row), respectively, from noisy data generated from the truncated cone-beam scan. First to fourth columns represent the 2-D slices in planes of $x=0, y=25 \mathrm{~mm}, z=0, z=6.4 \mathrm{~mm}$, respectively. Display window is [1.0, 1.04].

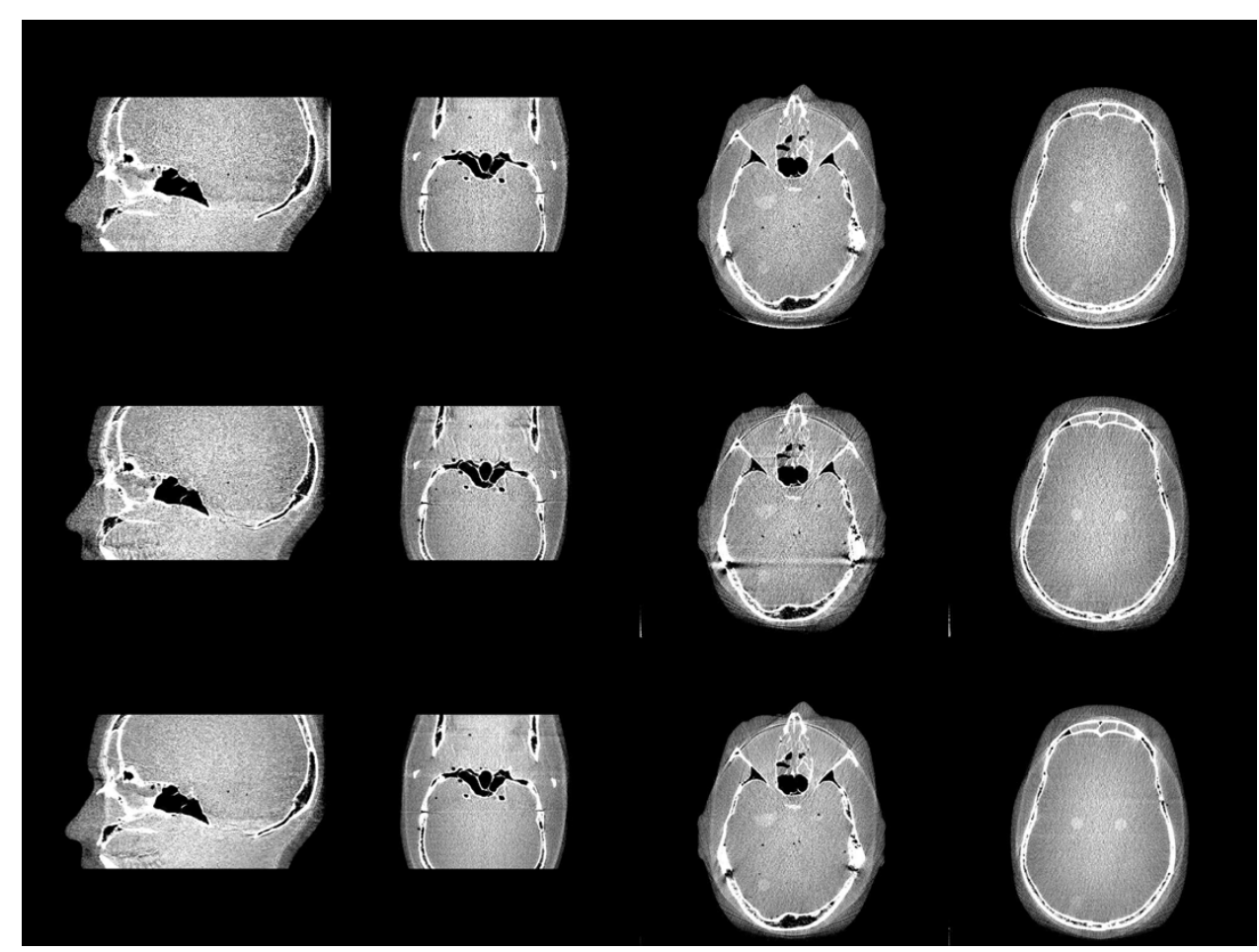

Fig. 11. Two-dimensional slices in 3-D images reconstructed by use of the FDK algorithm (upper row), the BPF algorithm in (13) (middle row), and the weighted BPF algorithm in (19) (lower row), respectively, from original data obtained from the Acuity cone-beam CT system. First to fourth columns represent the 2-D slices in planes of $x=0, y=0, z=0, z=-33.8 \mathrm{~mm}$, respectively. Display window is $[0.017,0.030] \mathrm{mm}^{-1}$.

There are potentially great practical benefits for the proposed BPF-based ROI-reconstruction algorithms for circular cone-beam CT. The proposed algorithms can aid in scans where, due to detector size constraints, some views are trun- cated. For example, this could occur in chest scanning where the shoulders might be truncated for some views. Another obvious advantage of the proposed algorithms is the potential application to dose reduction. Often times clinicians are only 


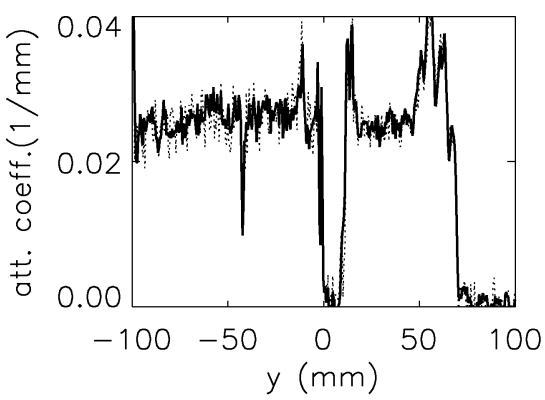

(a)

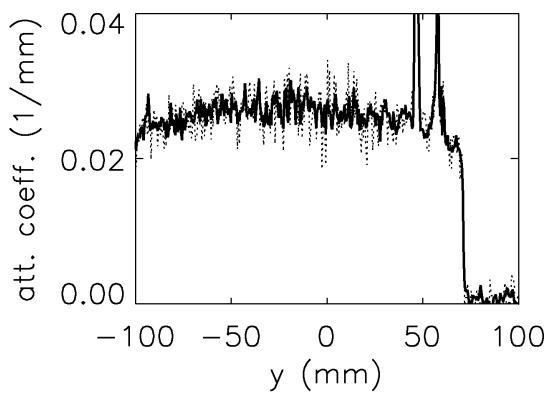

(b)

Fig. 12. Profiles in images displayed in Fig. 11 along (a) $z=0, x=0$ and (b) $z=-33.8 \mathrm{~mm}, x=0$. Dotted and solid curves represent the results obtained from the FDK and the proposed algorithm in (19), respectively.

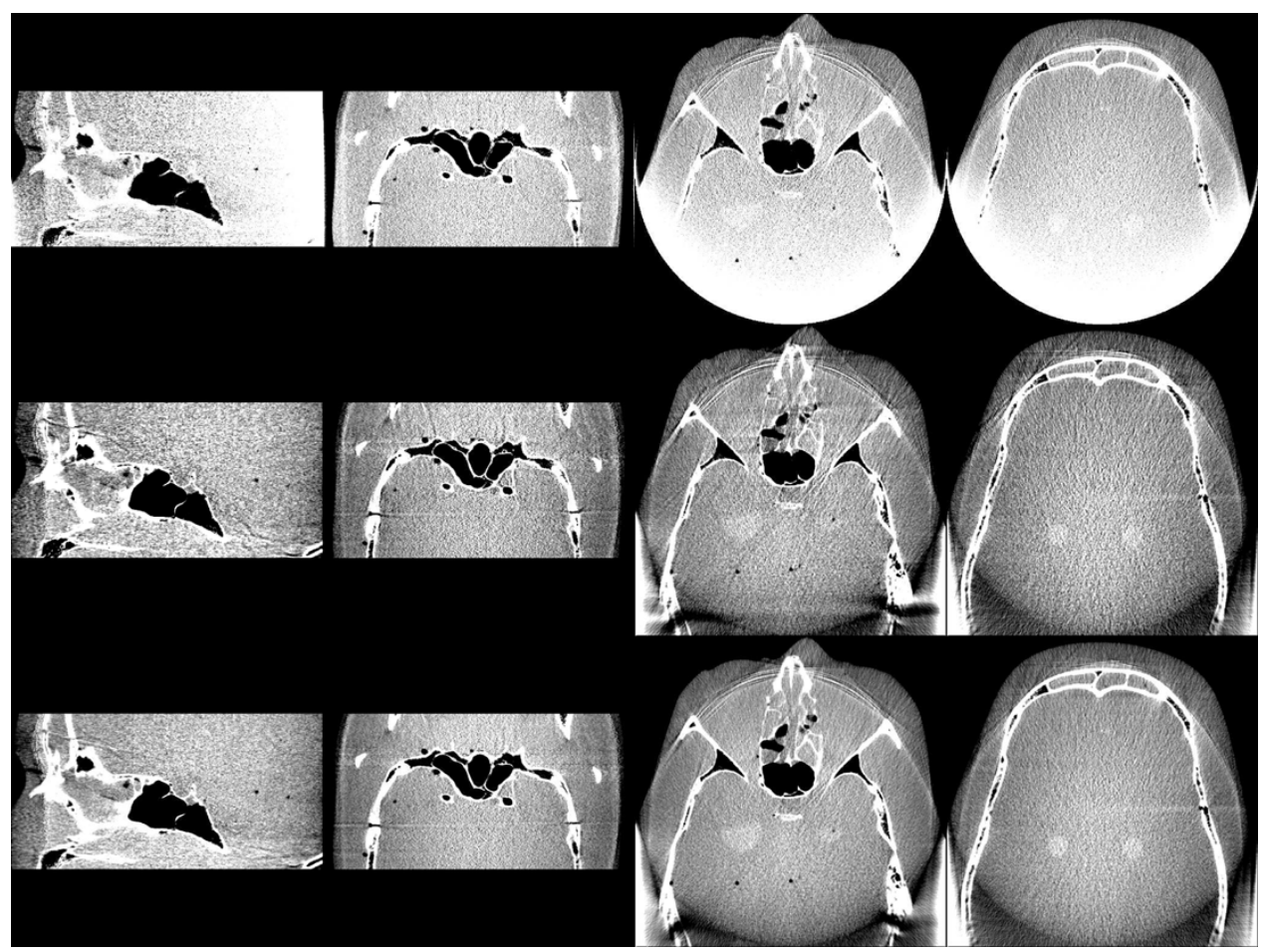

Fig. 13. Two-dimensional slices in 3-D images reconstructed by use of the FDK algorithm (upper row), the BPF algorithm in (13) (middle row), and the weighted BPF algorithm in (19) (lower row), respectively, from generated truncated data obtained from the Acuity cone-beam CT system. First to fourth columns represent the 2-D slices in planes of $x=0, y=0, z=0, z=-33.8 \mathrm{~mm}$, respectively. Display window is $[0.017,0.030] \mathrm{mm}^{-1}$.

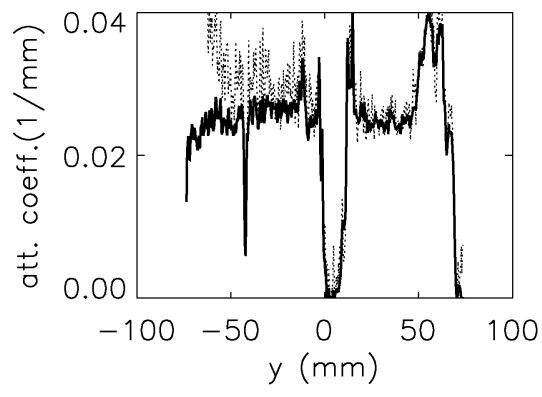

(a)

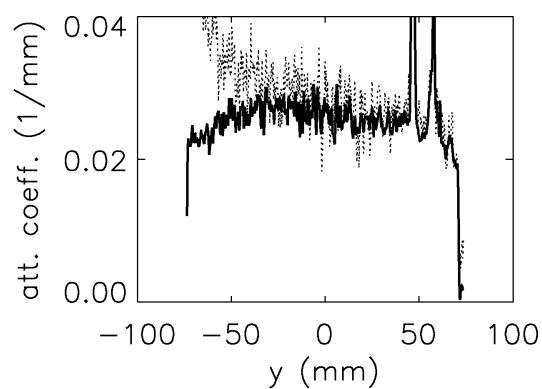

(b)

Fig. 14. Profiles in images displayed in Fig. 13 along (a) $z=0, x=0$ and (b) $z=-33.8 \mathrm{~mm}, x=0$. Dotted and solid curves represent the results obtained from the FDK and the proposed algorithm in (19), respectively.

interested in viewing a small subvolume of the patient. The algorithms presented here can allow the X-ray beam to be confined to exposing a smaller region of the body that includes the ROI. The reduced scanning angle of the ROI reconstruction algorithms for circular cone-beam CT can also reduce the required scanning time and radiation dose. 
APPENDIX I

DISCONTINUITY IN THE WEIGHTED BPF ALGORITHM

Substitution of $\omega(u, \lambda)=H_{0}(u, \lambda) \omega_{0}(u, \lambda)$ into (19) yields

$$
\begin{aligned}
g_{\pi c}^{(\omega)} & \left(x_{\pi}^{\prime}, \lambda_{1}, \lambda_{2}, z_{0}\right) \\
= & \int_{\lambda_{\min }}^{\lambda_{\max }} d \lambda \frac{S^{2}}{\left[R-\vec{r}^{\prime} \cdot \hat{\mathrm{e}}_{w}(\lambda)\right]^{2}} H_{0}(u, \lambda) \frac{\partial}{\partial u} \\
& \times\left[\frac{R}{A} \omega_{0}(u, \lambda) P(u, v, \lambda)\right] \\
& +\int_{\lambda_{\min }}^{\lambda_{\max }} d \lambda \frac{S^{2}}{\left[R-\vec{r}^{\prime} \cdot \hat{\mathrm{e}}_{w}(\lambda)\right]^{2}} \frac{R}{A} \omega_{0}(u, \lambda) P(u, v, \lambda) \\
& \times \frac{\partial}{\partial u} H_{0}(u, \lambda)+\left.\frac{\omega(u, \lambda) P(u, v, \lambda)}{\left|\vec{r}^{\prime}-\vec{r}_{c}(\lambda)\right|}\right|_{\lambda_{\min }} ^{\lambda_{\max }} .
\end{aligned}
$$

Noticing that (22) can be written as

$$
H_{0}(u, \lambda)=\operatorname{sgn}\left(\sin \left(\lambda-\arctan \frac{u}{S}-\theta\right)\right)
$$

one can express the derivative of the $H_{0}(u, \lambda)$ function as

$$
\begin{aligned}
\frac{\partial}{\partial u}\left[H_{0}(u, \lambda)\right]= & -2 \delta\left(\sin \left(\lambda-\arctan \frac{u}{S}-\theta\right)\right) \\
& \times \cos \left(\lambda-\arctan \frac{u}{S}-\theta\right) \cdot \frac{S}{u^{2}+S^{2}} .
\end{aligned}
$$

Using the expression of (26), one can rewrite the second term in (24) as

$$
\begin{aligned}
T_{2}= & -2 \int_{\lambda_{\min }}^{\lambda_{\max }} d \lambda \frac{S^{2}}{\left[R-\vec{r}^{\prime} \cdot \hat{\mathrm{e}}_{w}(\lambda)\right]^{2}} \frac{R}{A} \omega_{0}(u, \lambda) \\
& \times P(u, v, \lambda) \delta\left(\sin \left(\lambda-\arctan \frac{u}{S}-\theta\right)\right) \\
& \times \cos \left(\lambda-\arctan \frac{u}{S}-\theta\right) \cdot \frac{S}{u^{2}+S^{2}} .
\end{aligned}
$$

For a point $\left(x_{\pi}^{\prime}, \lambda_{1}, \lambda_{2}, z_{0}\right)$, on the PI-line segment, $\sin (\lambda-$ $\arctan (u / S)-\theta)=0$ has two solutions, which are $\lambda_{1}$ and $\lambda_{2}$. Considering the property of the $\delta$-function, one can re-express (27) as

$$
\begin{aligned}
T_{2}=-2 & \sum_{i=1}^{2} \frac{S^{2}}{\left[R-\vec{r}^{\prime} \cdot \hat{\mathrm{e}}_{w}\left(\lambda_{i}\right)\right]^{2}} \frac{R}{A} \omega_{0}\left(u, \lambda_{i}\right) P\left(u, v, \lambda_{i}\right) \\
& \times \frac{\cos \left(\lambda-\arctan \frac{u}{S}-\theta\right)}{\left|\frac{d}{d \lambda} \sin \left(\lambda-\arctan \frac{u}{S}-\theta\right)\right|_{\lambda=\lambda_{i}}} \cdot \frac{S}{u^{2}+S^{2}} .
\end{aligned}
$$

On the other hand, one can calculate

$$
\begin{aligned}
\frac{d u}{d \lambda} & =\frac{d}{d \lambda} \frac{S \vec{r}^{\prime} \cdot \hat{\mathrm{e}}_{u}(\lambda)}{R-\vec{r}^{\prime} \cdot \hat{\mathrm{e}}_{w}(\lambda)} \\
& =\frac{u^{2}+S^{2}}{S}-\frac{S R}{R-\vec{r}^{\prime} \cdot \hat{\mathrm{e}}_{w}(\lambda)} .
\end{aligned}
$$

Using this result in (28) yields

$$
\begin{aligned}
T_{2}= & -2 \sum_{i=1}^{2} \frac{S^{2}}{\left[R-\vec{r}^{\prime} \cdot \hat{\mathrm{e}}_{w}\left(\lambda_{i}\right)\right]^{2}} \frac{R}{A} \omega_{0}\left(u, \lambda_{i}\right) P\left(u, v, \lambda_{i}\right) \\
& \times \frac{\operatorname{sgn}\left(\cos \left(\lambda_{i}-\arctan \frac{u}{S}-\theta\right)\right)}{\left|\frac{S}{u^{2}+S^{2}} \frac{S R}{R-\vec{r}^{\prime} \cdot \hat{\mathrm{e}}_{w}(\lambda)}\right|_{\lambda=\lambda_{i}}} \cdot \frac{S}{u^{2}+S^{2}} \\
= & \left.\frac{2 \omega_{0}(u, \lambda) P(u, v, \lambda)}{\left|\vec{r}^{\prime}-\vec{r}_{c}(\lambda)\right|}\right|_{\lambda_{1}} ^{\lambda_{2}}
\end{aligned}
$$

in which we have invoked

$$
\begin{aligned}
& \cos \left(\lambda_{1}-\arctan \frac{u}{S}-\theta\right)=1 \\
& \cos \left(\lambda_{2}-\arctan \frac{u}{S}-\theta\right)=-1 .
\end{aligned}
$$

Finally, replacing the second term of (24) with (30), one obtains (23).

\section{ACKNOWLEDGMENT}

The authors would like to thank D. Xia for stimulating discussions.

\section{REFERENCES}

[1] E. L. Ritman, "Molecular imaging in small animals—roles for microCT," J. Cellular Biochem., vol. 39, pp. 116-124, 2002.

[2] N. L. Ford, M. M. Thomton, and D. W. Holdsworth, "Fundamental image quality limits for microcomputed tomography in small animals," Med. Phys., vol. 30, pp. 2869-2877, 2003.

[3] S.-C. Lee, H.-K. Kim, I.-K. Chun, M.-H. Cho, S.-Y. Lee, and M.-H. Cho, "A flat-panel detector based micro-CT system: Performance evaluation for small-animal imaging," Phys. Med. Biol., vol. 48, pp. 4173-4185, 2003.

[4] J. M. Boone, T. R. Nelson, K. K. Lindfors, and J. A. Seibert, "Dedicated breast CT: Radiation dose and image quality evaluation," Radiol., vol. 221, pp. 657-667, 2001

[5] B. Chen and R. Ning, "Cone-beam volume CT breast imaging: Feasibility study," Med. Phys., vol. 29, pp. 755-770, 2002.

[6] J. M. Boone, N. Shah, and T. R. Nelson, "A comprehensive analysis of $\mathrm{DgN}(\mathrm{CT})$ coefficients for pendant-geometry cone-beam breast computed tomography," Med. Phys., vol. 31, pp. 226-235, 2004.

[7] P. Cho, R. H. Johnson, and T. W. Griffin, "Cone-beam CT for radiotherapy applications," Phys. Med. Biol., vol. 40, pp. 1863-1883, 1995.

[8] D. A. Jaffray, J. H. Siewerdsen, J. W. Wong, and A. A. Martinez, "Flat-panel cone-beam computed tomography for image-guided radiation therapy," Int. J. Radiat. Oncol. Biol. Phys., vol. 53, pp. 1337-1349, 2002.

[9] E. C. Ford, J. Chang, K. Sidhu, D. Todor, G. Mageras, E. Yorke, C. C. Ling, and H. Amols, "Cone-beam CT with megavoltage beams and an amorphous silicon electronic portal imaging device: Potential for verification of radiotherapy of lung cancer," Med. Phys., vol. 29, pp. 2913-2924, 2002.

[10] Y. Cho, D. J. Moseley, J. H. Siewerdsen, and D. A. Jaffray, “Accurate technique for complete geometric calibration of cone-beam computed tomography systems," Med. Phys., vol. 32, pp. 968-983, 2005.

[11] H. K. Tuy, "An inversion formula for cone-beam reconstruction," SIAM J. Appl. Math., vol. 43, pp. 546-552, 1983.

[12] B. D. Smith, "Image reconstruction from cone-beam projections: Necessary and sufficient conditions and reconstruction methods," IEEE Trans. Med. Imag., vol. 4, pp. 14-25, 1985.

[13] L. A. Feldkamp, L. C. Davis, and J. W. Kress, "Practical cone-beam algorithm," J. Opt. Soc. Amer., vol. A1, pp. 612-619, 1984.

[14] H. Hu, "An improved cone-beam reconstruction algorithm for the circular orbit," Scanning, vol. 18, pp. 572-581, 1996.

[15] M. Grass, T. Köhler, and R. Proksa, "3-D cone-beam CT reconstruction for circular trajectories," Phys. Med. Biol., vol. 45, pp. 329-347, 2000.

[16] H. Turbell, "Cone-beam reconstruction using filtered backprojection," Ph.D. dissertation, Linköping Univ., Linköping, Sweden, 2001. 
[17] H. Kudo, F. Noo, M. Defrise, and R. Clackdoyle, "New super-shortscan algorithms for fan-beam and cone-beam reconstruction," in IEEE Nucl. Sci. Symp. Conf. Rec., 2002, vol. 2, pp. 902-906.

[18] L. Yu, X. Pan, and C. A. Pelizzari, "Image reconstruction with a shiftvariant filtration in circular cone-beam CT," Int. J. Imag. Sys. Tech., vol. 14, pp. 213-221, 2004.

[19] Y. Zou and X. Pan, "Exact image reconstruction on PI-line from minimum data in helical cone-beam CT," Phys. Med. Biol., vol. 49, pp. 941-959, 2004.

[20] _ _ "An extended data function and its backprojection onto PI-lines in helical cone-beam CT," Phys. Med. Biol., vol. 49, pp. N383-N387, 2004.

[21] A. Katsevich, "Theoretically exact FBP-type inversion algorithm for spiral CT," SIAM J. Appl. Math., vol. 62, pp. 2012-2026, 2002.

[22] F. Noo, R. Clackdoyle, and J. Pack, "A two-step Hilbert transform method for 2-D image reconstruction," Phys. Med. Biol., vol. 49, pp. 3903-3923, 2004.

[23] Y. Zou, X. Pan, and E. Y. Sidky, "Image reconstruction in regions-ofinterest from truncated projections in a reduced fan-beam scan," Phys. Med. Biol., vol. 50, pp. 13-27, 2005.

[24] X. Pan, Y. Zou, and D. Xia, "Peripheral and central ROI-image reconstruction from and data-redundancy exploitation in truncated fan-beam data," Med. Phys., vol. 32, pp. 673-684, 2005.

[25] P. E. Danielsson, P. Edholm, and M. Seger, "Toward exact 3-D-reconstruction for helical cone-beam scanning of long objects. A new detector arrangement and a new completeness condition," in Proc. 1997 Int. Meeting Fully Three-Dimensional Image Reconstruction Radiol. Nucl. Med., D. W. Townsend and P. E. Kinahan, Eds., Pittsburgh, PA, 1997, pp. 141-144.
[26] M. Defrise, F. Noo, and H. Kudo, "A solution to the long-object problem in helical cone-beam tomography," Phys. Med. Biol., vol. 45, pp. 623-643, 2000

[27] Y. Zou and X. Pan, "Image reconstruction on PI-lines by use of filtered backprojection in helical cone-beam CT," Phys. Med. Biol., vol. 49, pp. 2717-2731, 2004.

[28] E. Y. Sidky, Y. Zou, and X. Pan, "Minimum data image reconstruction algorithms with shift-invariant filtering for helical, cone-beam CT," Phys. Med. Biol., vol. 50, pp. 1643-1657, 2005.

[29] X. Pan, D. Xia, Y. Zou, and L. Yu, "A unified analysis of FBP-based algorithms in helical cone-beam and circular cone- and fan-beam scans," Phys. Med. Biol., vol. 49, pp. 4349-4369, 2004.

[30] D. L. Parker, "Optimal short scan convolution reconstruction for fanbeam CT," Med. Phys., vol. 9, pp. 245-257, 1982.

[31] L. Yu and X. Pan, "Halfscan fan-beam computed tomography with improved noise and resolution properties," Med. Phys., vol. 30, pp. 2629-2637, 2003.

[32] Y. Zou, X. Pan, and E. Y. Sidky, "Theory and algorithms for image reconstruction on chords and within region of interests," J. Opt. Soc. Amer. A, vol. 22, pp. 272-2384, 2005. 